Article

\title{
Vasorelaxant and Antioxidant Activities of Spilanthes acmella Murr.
}

Orapin Wongsawatkul $^{1}$, Supaluk Prachayasittikul ${ }^{2, *}$, Chartchalerm Isarankura-Na-Ayudhya ${ }^{3}$, Jutamaad Satayavivad ${ }^{4}$, Somsak Ruchirawat ${ }^{5}$ and Virapong Prachayasittikul ${ }^{3, *}$

1 Department of Pharmacology, Faculty of Medicine, Srinakharinwirot University, Bangkok 10110, Thailand. E-Mail: orapinw@swu.ac.th

2 Department of Chemistry, Faculty of Science, Srinakharinwirot University, Bangkok 10110, Thailand

3 Department of Clinical Microbiology, Faculty of Medical Technology, Mahidol University, Bangkok 10700, Thailand. E-Mail: mtcis@mahidol.ac.th

4 Laboratory of Pharmacology, Chulabhorn Research Institute, Bangkok 10210, Thailand.

E-Mail: jutamaad@cri.or.th

5 Laboratory of Medicinal Chemistry, Chulabhorn Research Institute, Bangkok 10210, Thailand. E-Mail:somsak@cri.or.th

* Authors to whom correspondence should be addressed;

E-Mail: supaluk@swu.ac.th (S. P.); Tel. 662-664-1000 ext. 8209; Fax: 662-259-2097;

E-Mail: mtvpr@mahidol.ac.th (V. P.); Tel.662-418-0227; Fax: 662-412-4110

Received: 12 June 2008; in revised form: 14 December 2008 / Accepted: 18 December 2008 /

Published: 18 December 2008

Abstract: This study reports the effect of Spilanthes acmella Murr. extracts on phenylephrine-induced contraction of rat thoracic aorta as well as their antioxidant activity. Results show that the extracts exert maximal vasorelaxations in a dose-dependent manner, but their effects are less than acetylcholine-induced nitric oxide (NO) vasorelaxation. Significant reduction of vasorelaxations is observed in both $N^{\mathrm{G}}$-nitro-L-arginine methyl ester (L-NAME) and indomethacin (INDO). In the presence of L-NAME plus INDO, synergistic effects are observed, leading to loss of vasorelaxation of both acetylcholine and the extracts. Similarly, the vasorelaxations of the extracts are completely abolished upon the removal of endothelial cells. This demonstrates that the extracts exhibit vasorelaxation 
via partially endothelium-induced NO and prostacyclin in a dose-dependent manner. Significantly, the ethyl acetate extract exerts immediate vasorelaxation (ED $5076.1 \mathrm{ng} / \mathrm{mL}$ ) and is the most potent antioxidant (DPPH assay). The chloroform extract shows the highest vasorelaxation and antioxidation (SOD assay). These reveal a potential source of vasodilators and antioxidants.

Keywords: Spilanthes acmella Murr., vasorelaxant, antioxidant activities, nitric oxide, prostacyclin, thoracic aorta.

\section{Introduction}

Spilanthes acmella Murr. (para-cress or toothache plant) is a medicinal plant of the Compositae family [1]. It is known in Thai as Phak-Kratt Huawaen and has long been used as a traditional medicine for toothache, headache and treatment of asthma, rheumatism, fever, sore throat and haemorrhoids [1-3]. Its root decoction has been used as a laxative and diuretic drug [2, 4]. It is an annual or short-lived herb, spreads in low open places and requires moist soil [5]. Its flowers and leaves have a pungent taste, accompanied with tingling and numbness [2].

Constituents found in Spilanthes acmella Murr. were spilanthol, isobutylamide derivatives [6, 7], $\alpha$ and $\beta$-amyrin esters, stigmasterol, myricyl alcohol including sitosterol glucosides [8] and triterpenoidal saponins [9]. Spilanthol showed interesting bioactivities, e.g. strong local anesthetic [10], analgesic [11, 12] and insecticidal activities [3]. Crude flower head extracts of the plant exhibited potent ovicidal, marked larvicidal ( $\mathrm{LC}_{50}$ of $61.43 \mathrm{ppm}$ ) and pupicidal activities [13, 14]. The extracts were also shown to exhibit pancreatic lipase inhibition which has potential as candidates for weight loss and obesity control [15]. In addition, the crude flower head extracts displayed strong diuretic action which caused marked increase in urine $\mathrm{Na}^{+}, \mathrm{K}^{+}$and a reduction in the osmolarity of urine [16]. Moreover, it has been used as anti-toothache formulation for pain relieving within 2-10 min and also useful in treating the swelling and infection of gums [17]. The Spilanthes acmella is one of the active ingredients in compositions for acute- or long-term treatment of microbial infections, particularly, oral pathogenic microorganisms, dental caries, periodontosis, gum disease, gum bleeding and/or plaque reduction [18]. In addition, its root powder extract is very effective in treating HIV/AID infection [19]. Presently, cardiovascular disease is one of the most important public health concerns, accountable for morbidity and mortality. Thus new vasoactive ingredients that influence the endothelial cells, which are key regulating cells in the vessel wall, are the striking areas. So far, the effect of Spilanthes acmella Murr. on vascular function has not been reported. In this regard, it was of great interest to explore its medicinal value with regards to cardiovascular functions such as regulation of vascular tone. In the present study, we have investigated the effects of Spilanthes acmella Murr. extracts on rat thoracic aorta and its mechanism of action as well as its antioxidant activity. 


\section{Results}

\subsection{Components and HPLC (high performance liquid chromatography) profiles of the extracts}

Preliminary screening of components in the Spilanthes acmella Murr. extracts was performed using thin layer chromatography (TLC) chromatograms, IR and ${ }^{1} \mathrm{H}-\mathrm{NMR}$ spectra. It was found that hexane, chloroform, ethyl acetate and methanol extracts showed UV absorption on TLC. The ethyl acetate and methanol extracts exhibited very strong absorptions, compared to the chloroform extract. The IR spectra of the extracts showed absorptions $\left(\mathrm{cm}^{-1}\right)$ corresponding to $\mathrm{OH}$ (3360-3385), CH (2850-2942), CO (1710-1736), 1454-1574 and C-O (1047-1385) functions. The ethyl acetate and methanol extracts exhibited very strong $\mathrm{OH}$ and $\mathrm{CO}$ (ester or acid) and strong C-O absorptions. The chloroform extract displayed strong $\mathrm{OH}$, medium $\mathrm{CO}$ (ester or acid) and $\mathrm{C}-\mathrm{O}$ absorptions. The hexane extract showed strong $\mathrm{CO}$ (ester or acid), but medium $\mathrm{OH}$ and weak $\mathrm{C}-\mathrm{O}$ absorptions. All the extracts showed strong $\mathrm{CH}$ absorptions, the the hexane extract displaying the strongest ones. The ${ }^{1} \mathrm{H}-\mathrm{NMR}$ spectra of the hexane and chloroform extracts exhibited the presence of triterpenes $(\delta 0.9-2.4,3.4-3.7,4.1-4.3$ and 5.3-5.4). The ethyl acetate extract showed the signals of triterpenes and aromatics ( $\delta 0.84-2.4,3.5-4.3$, 5.3-5.5 and 6.8-7.8). The methanol extract displayed the presence of aromatic protons ( $\delta 6.8-8.4)$.

Based on the IR and ${ }^{1} \mathrm{H}-\mathrm{NMR}$ data, it may be concluded that the hexane and chloroform extracts contain triterpenes and long chain hydrocarbon esters or alcohols. The ethyl acetate extract contains triterpene esters and phenolic compounds, while the components of the methanol extract are presumably phenolic compounds and esters.

Profiles of the extracts were obtained by reversed phase HPLC running from a more polar to less polar mobile phase (Figure 1). Major nonpolar components of the extracts appeared in the 48.80-58.38 min retention time (RT) range. In the hexane extract the major component (66.06\%) was found at a RT of $52.56 \mathrm{~min}$, while th major components of the chloroform (65.72\%), ethyl acetate (67.34\%) and methanol (64.18\%) extracts were observed at RTs of 52.54, 52.58 and 52.60 min, respectively. It was noted that minor contents (0.22-4.72\%) of polar components in chloroform and ethyl acetate extracts appeared at RTs of 18.27-33.73 min. However, the methanol extract showed a minor polar component (2.64\%) at a RT of $23.82 \mathrm{~min}$ (Table 1).

\subsection{Vasorelaxant activity}

Effects of Spilanthes acmella Murr. extracts (hexane, chloroform, ethyl acetate and methanol) on vascular function of rat thoracic aorta precontracted with phenylephrine (PE) were investigated under various conditions; in the presence or absence of inhibitors, namely, $N^{\mathrm{G}}$-nitro-L-arginine methyl ester (L-NAME) and indomethacin (INDO) and under removal of functional endothelial cells. In addition, the effects of acetylcholine (ACh) as a positive control, sodium nitroprusside (SNP) a negative control and vehicle such as polyethyleneglycol (PEG) were studied. Results confirmed that the vasorelaxation of ACh was related to nitric oxide (NO). 
Figure 1. HPLC profiles of plant extracts in hexane (a, $228.0 \mathrm{~nm}$ ), chloroform (b, 228.0 $\mathrm{nm}$ ), ethyl acetate (c, $228.0 \mathrm{~nm})$, and methanol (d, $280.0 \mathrm{~nm})$.

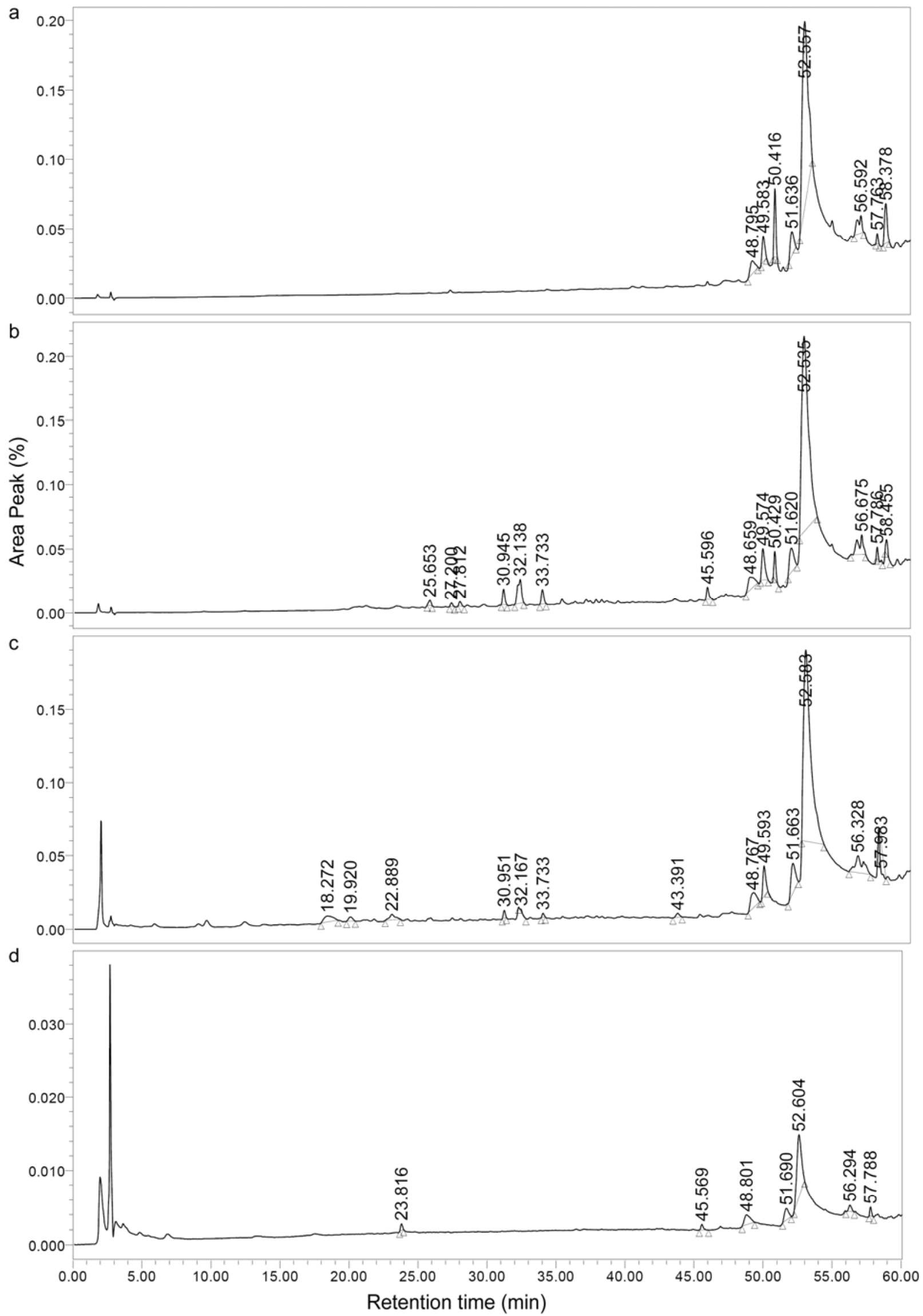


Table 1. Retention times and area peaks of plant extracts.

\begin{tabular}{|c|c|c|c|c|c|}
\hline Extract & $\begin{array}{c}\text { RT } \\
\text { (min) }\end{array}$ & $\begin{array}{c}\text { Area } \\
(\%)\end{array}$ & Extract & $\begin{array}{c}\text { RT } \\
\text { (min) }\end{array}$ & $\begin{array}{c}\text { Area } \\
(\%)\end{array}$ \\
\hline \multirow[t]{9}{*}{ Hexane } & 48.80 & 3.86 & Chloroform $^{\mathrm{b}}$ & 25.65 & 0.61 \\
\hline & 49.58 & 4.20 & & 27.20 & 0.37 \\
\hline & 50.42 & 7.79 & & 27.81 & 0.55 \\
\hline & 51.64 & 5.38 & & 30.95 & 1.69 \\
\hline & 52.56 & 66.06 & & 32.14 & 4.72 \\
\hline & 56.59 & 5.09 & & & \\
\hline & 57.76 & 0.97 & Ethyl acetate ${ }^{\mathrm{b}}$ & 18.27 & 2.55 \\
\hline & 58.38 & 6.64 & & 19.92 & 0.84 \\
\hline & & & & 22.89 & 1.57 \\
\hline \multirow[t]{2}{*}{ Chloroform $^{\mathrm{a}}$} & 52.54 & 65.72 & & 30.95 & 0.72 \\
\hline & & & & 32.17 & 0.22 \\
\hline Ethyl acetate $^{a}$ & 52.58 & 67.34 & & 33.73 & 0.38 \\
\hline Methanol $^{\mathrm{a}}$ & 52.60 & 64.18 & Methanol $^{\mathrm{b}}$ & 23.82 & 2.64 \\
\hline
\end{tabular}

${ }^{a}$ Minor contents at RT range 48-58 min are similar to hexane extract, but data are not shown.

${ }^{\mathrm{b}}$ Minor contents of polar components at RT range 18.27-33.73 min.

2.3. Effect of Spilanthes acmella Murr. extracts on the vascular function of rat thoracic aorta in the absence or presence of a NOS inhibitor (L-NAME, $1 \mathrm{mM})$

\subsubsection{Hexane extract}

In the rat aortic ring preparation, the hexane extract exerted vasorelaxation in the dose-dependent manner, but PEG showed no effect on the vessels (Figure 2 and Table 2). The activity of the hexane extract reached up to $65.7 \%$ of the $\mathrm{R}_{\max }$ induced by ACh $(110.1 \%)$ showing $\mathrm{ED}_{50} 3.60 \times 10^{-7} \mathrm{mg} / \mathrm{mL}$ and $4.66 \times 10^{-7} \mathrm{M}$, respectively. This indicated that the hexane extract likely acted as a partial agonist. In the presence of a NOS inhibitor (L-NAME), the dose-response curve of hexane extract was shifted to the right, with $\mathrm{R}_{\max } 33.5 \%$ and $\mathrm{ED}_{50} 4.80 \times 10^{-7} \mathrm{mg} / \mathrm{mL}$. This suggested that the hexane extract caused vasorelaxation by partially producing NO from the endothelial cells, while PEG had no effect on induction of vasorelaxation.

\subsubsection{Chloroform extract}

Similarly, the chloroform extract showed dose-dependent vasorelaxation (Figure 3 and Table 2). The $\mathrm{R}_{\max }$ of $\mathrm{ACh}$ and the chloroform extract were 120.6 and $96.6 \%$, with $\mathrm{ED}_{50} 9.43 \times 10^{-7} \mathrm{M}$ and $4.28 \times 10^{-7} \mathrm{mg} / \mathrm{mL}$, respectively. In the presence of L-NAME, the dose-response curve of chloroform extract was shifted to the right. The extract showed $R_{\max } 54.4 \%$ and $\mathrm{ED}_{50} 1.00 \times 10^{-6} \mathrm{mg} / \mathrm{mL}$, whereas $\mathrm{R}_{\max }$ and $\mathrm{ED}_{50}$ of $\mathrm{ACh}$ were $82.1 \%$ and $3.61 \times 10^{-7} \mathrm{M}$, respectively. This demonstrated that the chloroform extract exhibited vasorelaxation by partly production of NO from the endothelial cells. 
Table 2. Vasorelaxant activity of Spilanthes acmella Murr. extracts on rat thoracic aorta.

\begin{tabular}{|c|c|c|c|c|}
\hline \multirow{3}{*}{ Compound } & \multicolumn{4}{|c|}{ Vasorelaxant activity } \\
\hline & \multicolumn{2}{|c|}{ Without L-NAME } & \multicolumn{2}{|c|}{ With L-NAME (1 mM) } \\
\hline & $\mathbf{R}_{\max }(\%)$ & $\mathrm{ED}_{50}(\mathrm{mg} / \mathrm{mL})$ & $\mathbf{R}_{\max }(\%)$ & $\mathrm{ED}_{50}(\mathrm{mg} / \mathrm{mL})$ \\
\hline Hexane extract $^{\mathrm{a}}$ & $65.67 \pm 0.984$ & $3.601 \times 10^{-7}$ & $33.49 \pm 1.122$ & $4.805 \times 10^{-7}$ \\
\hline $\mathrm{ACh}^{\mathrm{a}}$ & $110.08 \pm 1.801$ & $4.657 \times 10^{-7}$ & NA & NA \\
\hline Chloroform extract $^{\mathrm{b}}$ & $96.64 \pm 1.112$ & $4.279 \times 10^{-7}$ & $54.38 \pm 0.575$ & $1.003 \times 10^{-6}$ \\
\hline $\mathrm{ACh}^{\mathrm{a}}$ & $120.63 \pm 1.671$ & $9.428 \times 10^{-7}$ & $82.15 \pm 1.158$ & $3.611 \times 10^{-7}$ \\
\hline Ethyl acetate extract ${ }^{\mathrm{b}}$ & $81.64 \pm 0.530$ & $7.614 \times 10^{-8}$ & $53.99 \pm 1.517$ & $3.885 \times 10^{-7}$ \\
\hline $\mathrm{ACh}^{\mathrm{b}}$ & $115.47 \pm 0.951$ & $5.912 \times 10^{-7}$ & $83.36 \pm 1.169$ & $5.650 \times 10^{-7}$ \\
\hline Methanol extract ${ }^{\mathrm{a}}$ & $65.09 \pm 0.409$ & $9.550 \times 10^{-7}$ & $33.38 \pm 0.403$ & $1.375 \times 10^{-6}$ \\
\hline $\mathrm{ACh}^{\mathrm{a}}$ & $120.69 \pm 0.220$ & $8.183 \times 10^{-7}$ & $82.01 \pm 0.582$ & $3.427 \times 10^{-7}$ \\
\hline $\mathrm{ACh}^{\mathrm{b}}$ & $119.20 \pm 0.344$ & $7.878 \times 10^{-7}$ & $80.86 \pm 0.368$ & $4.163 \times 10^{-7}$ \\
\hline $\mathrm{SNP}^{\mathrm{b}}$ & $120.53 \pm 2.270$ & $1.727 \times 10^{-7}$ & $114.84 \pm 0.716$ & $3.171 \times 10^{-7}$ \\
\hline
\end{tabular}

a: data obtained from 6 experiments, b: data obtained from 5 experiments

$\mathrm{ED}_{50}$ of ACh and SNP are expressed as molar, NA: not tested, $p<0.05$

Figure 2. Effect of hexane extract on the vascular function of rat thoracic aorta in the absence or presence of NOS inhibitor (L-NAME, $1 \mathrm{mM}$ ) compared with those of ACh and PEG.

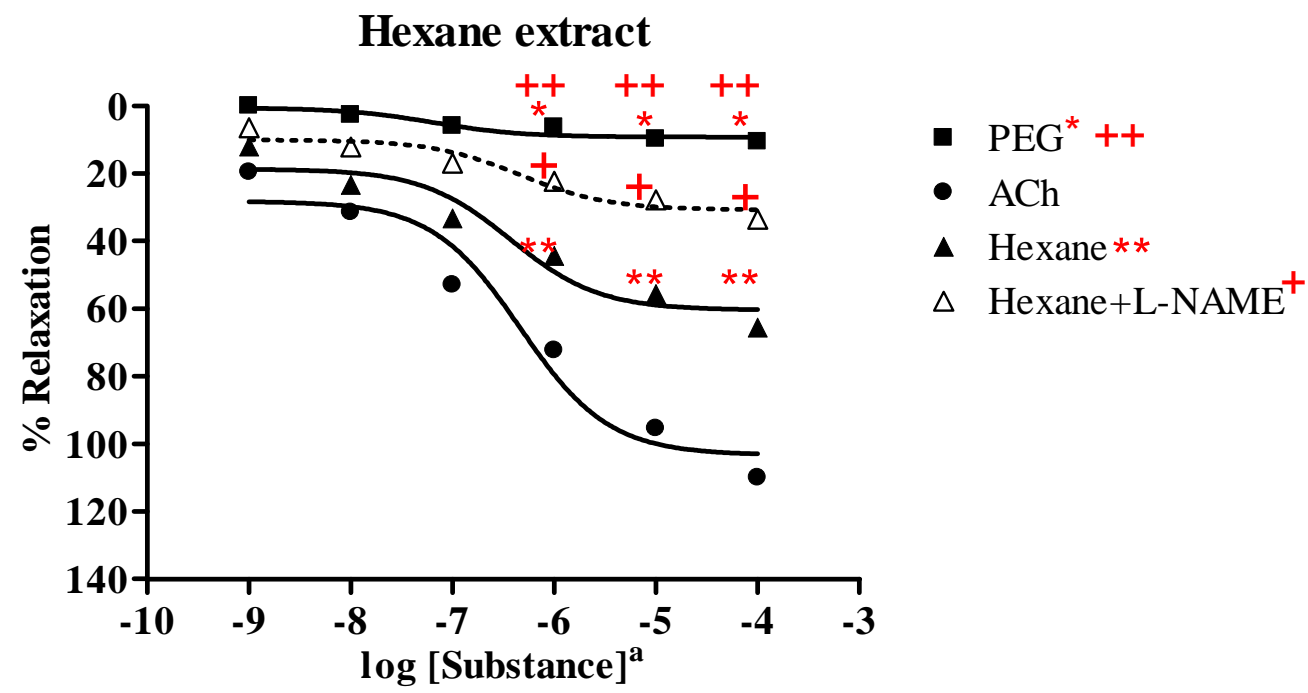

Data represent as means \pm s.e.m. of 6 experiments, each performed in duplicate.

$\star p<0.05$, ACh versus PEG,

$\star \star p<0.05$, ACh versus hexane extract,

$+p<0.05$, hexane extract versus hexane extract + L-NAME,

$++p<0.05$, PEG versus hexane extract

a: hexane extract as $\mathrm{mg} / \mathrm{mL}$, ACh as molar 


\subsubsection{Ethyl acetate extract}

A similar result was obtained from ethyl acetate extract, showing vasorelaxation in the dosedependent manner (Figure 4 and Table 2). The ACh and plant extract exerted $\mathrm{R}_{\max } 115.5$ and 81.6\%, with $\mathrm{ED}_{50}$ of $5.91 \times 10^{-7} \mathrm{M}$ and $7.61 \times 10^{-8} \mathrm{mg} / \mathrm{mL}$, respectively. This showed that the ethyl acetate extract possibly acted as a partial agonist. In the presence of L-NAME, the dose-response curve of the extract was shifted to the right with $\mathrm{R}_{\max } 54.0 \%, \mathrm{ED}_{50} 3.88 \times 10^{-7} \mathrm{mg} / \mathrm{mL}$, whereas the $\mathrm{R}_{\max }$ and $\mathrm{ED}_{50}$ of ACh were $83.4 \%$ and $5.65 \times 10^{-7} \mathrm{M}$, respectively. This suggested that the ethyl acetate extract caused vasorelaxation by partly synthesis of NO from the endothelial cells.

\subsubsection{Methanol extract}

The methanol extract displayed vasorelaxant activity in a dose-dependent manner (Figure 5 and Table 2). The $\mathrm{R}_{\max }$ of $\mathrm{ACh}$ and extract were 120.7 and $65.1 \%$ with $\mathrm{ED}_{50} 8.18 \times 10^{-7} \mathrm{M}$ and $9.55 \times 10^{-7} \mathrm{mg} / \mathrm{mL}$, respectively. This indicated that the extract possibly acted as partial agonist. In the presence of L-NAME, the dose-response curve of extract was shifted to the right with $\mathrm{R}_{\max } 33.4 \%$ and $\mathrm{ED}_{50} 1.38 \times 10^{-6} \mathrm{mg} / \mathrm{mL}$, whereas the $\mathrm{R}_{\max }$ and $\mathrm{ED}_{50}$ of ACh were $82.0 \%$ and $3.43 \times 10^{-7} \mathrm{M}$, respectively. This illustrated that the methanol extract exerted vasorelaxation by partial NO production from the endothelial cells.

Figure 3. Effect of $\mathrm{CHCl}_{3}$ extract and $\mathrm{ACh}$ on the vascular function of rat thoracic aorta in the absence or presence of $1 \mathrm{mM}$ L-NAME.

\section{$\mathrm{CHCl}_{3}$ extract}

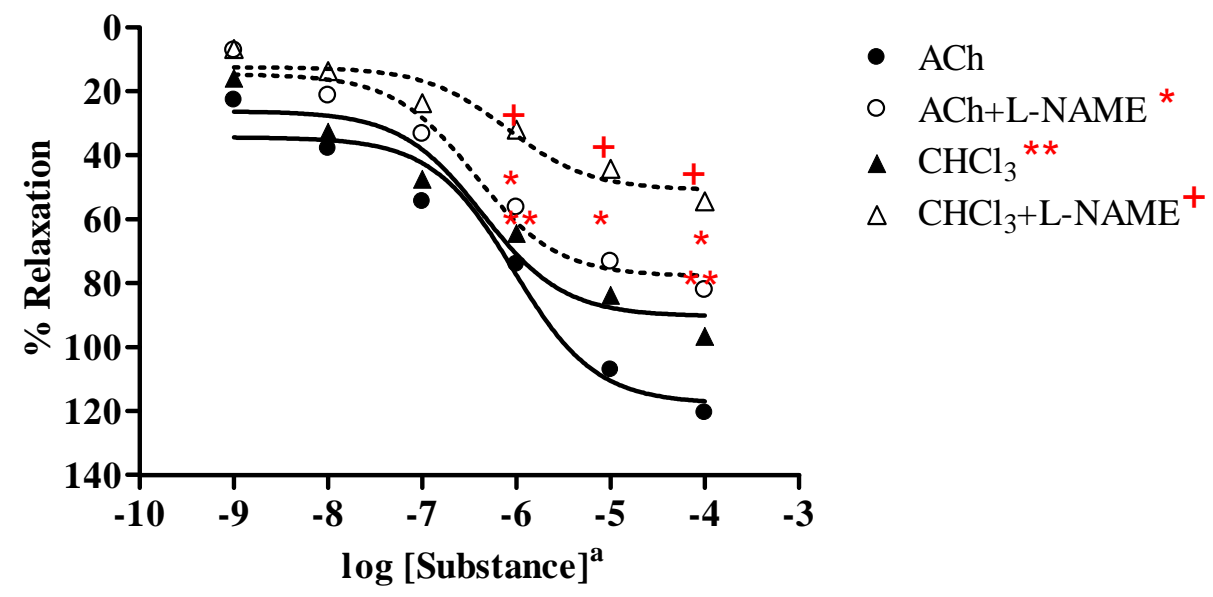

Data represent as means \pm s.e.m. of 5-6 experiments, each performed in duplicate.

$\star p<0.05$, ACh versus ACh+L-NAME,

$\star \star p<0.05, \mathrm{ACh}$ versus $\mathrm{CHCl}_{3}$ extract,

$+p<0.05, \mathrm{CHCl}_{3}$ extract versus $\mathrm{CHCl}_{3}$ extract+L-NAME

a: $\mathrm{CHCl}_{3}$ extract as $\mathrm{mg} / \mathrm{mL}$, ACh as molar 
Figure 4. Effect of EtOAc extract and ACh on the vascular function of rat thoracic aorta in the absence or presence of $1 \mathrm{mM}$ L-NAME.

\section{EtOAc extract}

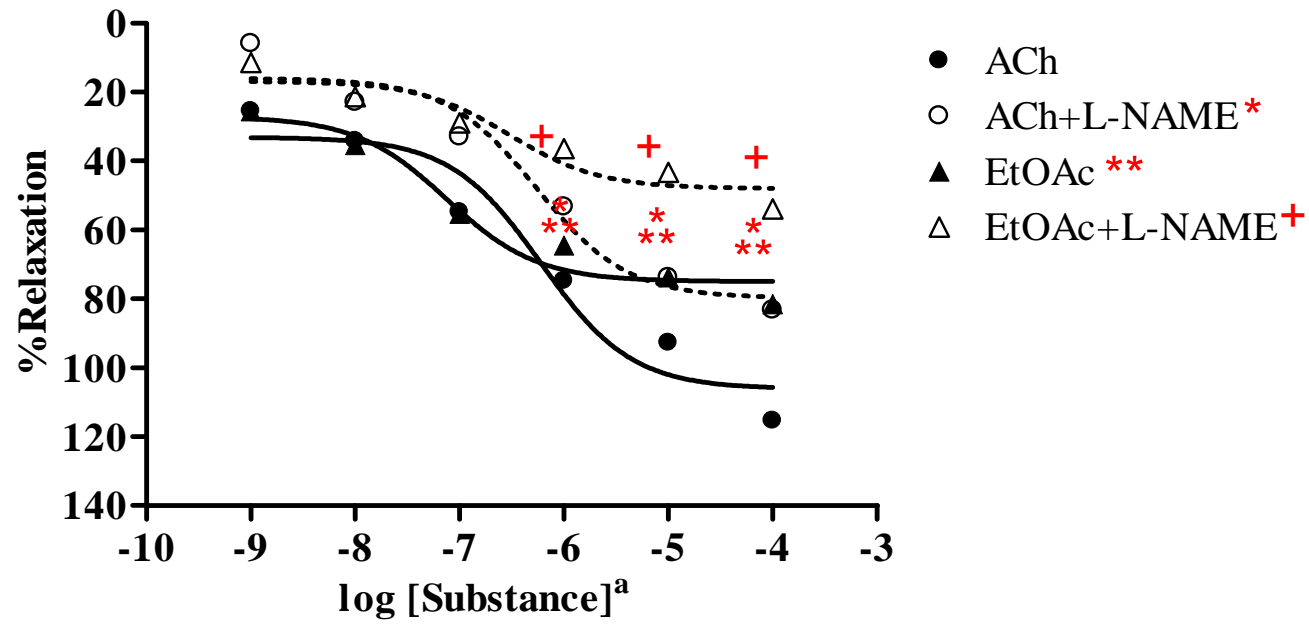

Data represent as means \pm s.e.m. of 5 experiments, each performed in duplicate.

$\star p<0.05$, ACh versus ACh+L-NAME,

$\star \star p<0.05$, ACh versus EtOAc extract,

$+p<0.05$, EtOAc extract versus EtOAc extract+L-NAME

a: EtOAc extract as $\mathrm{mg} / \mathrm{mL}$, ACh as Molar

Figure 5. Effect of $\mathrm{MeOH}$ extract and $\mathrm{ACh}$ on the vascular function of rat thoracic aorta in the absence and presence of $1 \mathrm{mM}$ L-NAME.

\section{MeOH extract}

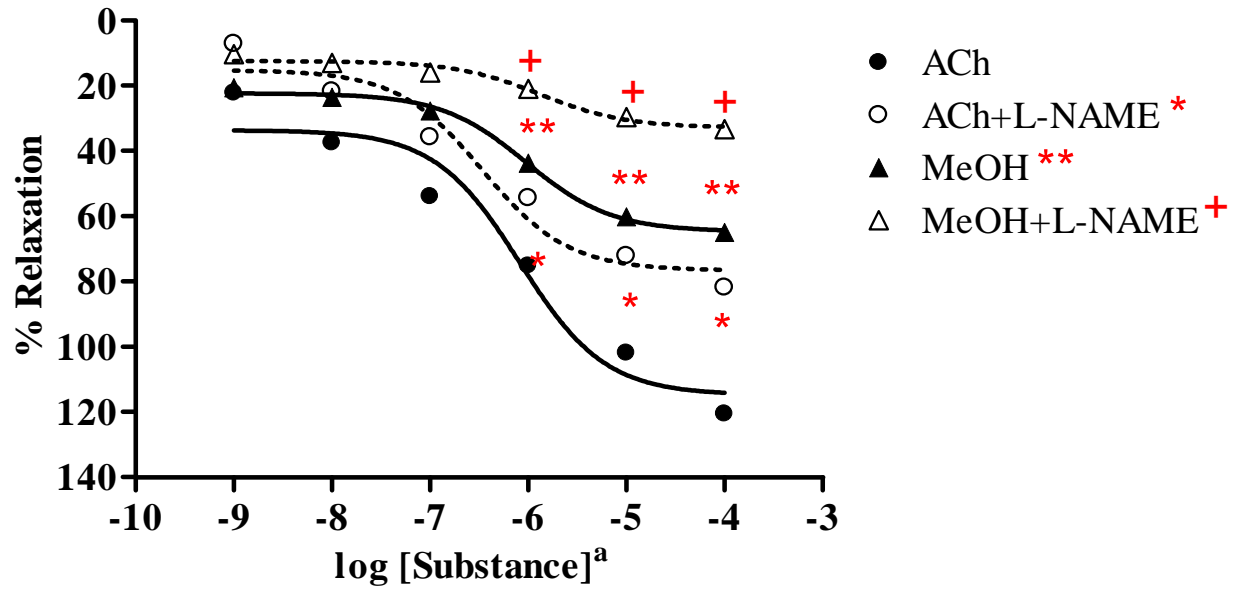

Data represent as means \pm s.e.m. of 6 experiments, each performed in duplicate.

$\star p<0.05$, ACh versus ACh+L-NAME,

$\star \star p<0.05$, ACh versus $\mathrm{MeOH}$ extract,

$+p<0.05, \mathrm{MeOH}$ extract versus $\mathrm{MeOH}$ extract+L-NAME

a: $\mathrm{MeOH}$ extract as $\mathrm{mg} / \mathrm{mL}$, ACh as molar 
2.4. Effect of Spilanthes acmella Murr. on the vascular function of rat thoracic aorta in the absence of endothelial cells

The vasorelaxation of Spilanthes acmella Murr. extracts was examined with and without intact endothelial cells, comparing with ACh. The results (Table 3) illustrated that the vasorelaxation of all the tested extracts was abolished when endothelial cells were removed. An example, the dose-response curve of the ethyl acetate extract is shown in Figure 6. Similar results were observed for the control, ACh. This confirmed that the vasorelaxant activity of Spilanthes acmella Murr. extracts was mediated via endothelial cells producing NO.

Table 3. Effect of endothelial cells on vasorelaxant activity of Spilanthes acmella Murr. extracts.

\begin{tabular}{|c|c|c|c|c|}
\hline \multirow{3}{*}{ Compound $^{a}$} & \multicolumn{4}{|c|}{ Vasorelaxant activity } \\
\hline & \multicolumn{2}{|c|}{$+\mathbf{E t}$} & \multicolumn{2}{|c|}{$-\mathbf{E t}$} \\
\hline & $\mathbf{R}_{\max }(\%)$ & $\mathrm{ED}_{50}(\mathrm{mg} / \mathrm{mL})$ & $\mathbf{R}_{\max }(\%)$ & $\mathrm{ED}_{50}(\mathrm{mg} / \mathrm{mL})$ \\
\hline Hexane extract & $64.82 \pm 0.870$ & $2.556 \times 10^{-7}$ & 0 & - \\
\hline $\mathrm{ACh}$ & $104.31 \pm 1.595$ & $2.440 \times 10^{-7}$ & $7.16 \pm 2.010$ & $3.128 \times 10^{-7}$ \\
\hline Chloroform extract & $98.00 \pm 0.694$ & $4.422 \times 10^{-7}$ & $5.64 \pm 0.080$ & $3.128 \times 10^{-7}$ \\
\hline $\mathrm{ACh}$ & $120.36 \pm 0.823$ & $7.534 \times 10^{-7}$ & $7.42 \pm 0.159$ & $3.128 \times 10^{-7}$ \\
\hline Ethyl acetate extract & $81.68 \pm 0.682$ & $7.638 \times 10^{-8}$ & 0 & - \\
\hline $\mathrm{ACh}$ & $118.58 \pm 0.550$ & $7.477 \times 10^{-7}$ & $7.42 \pm 0.159$ & $3.128 \times 10^{-7}$ \\
\hline Methanol extract & $65.62 \pm 0.651$ & $1.002 \times 10^{-6}$ & 0 & - \\
\hline $\mathrm{ACh}$ & $119.93 \pm 0.495$ & $7.967 \times 10^{-7}$ & $7.28 \pm 0.138$ & $3.128 \times 10^{-7}$ \\
\hline
\end{tabular}

2.5. Effect of Spilanthes acmella Murr. on the vascular function of rat thoracic aorta in the presence of a cyclooxygenase inhibitor (INDO)

The vasorelaxation of Spilanthes acmella Murr. extracts was studied in the presence of INDO (1 mM), comparing with L-NAME (1 mM) and L-NAME plus INDO. The results are summarized in Table 4 and some dose-response curves are shown in Figures 7-9. It was observed that in each experiment with L-NAME $(1 \mathrm{mM})$ or INDO $(1 \mathrm{mM})$ the vasorelaxation was reduced in a dosedependent manner. The inhibition effect of the INDO was stronger than that of L-NAME. However, the antagonist effects of L-NAME or INDO were more pronounced in the case of L-NAME plus INDO. Such effects were not observed in the SNP dose-response curves. Interestingly, the vasorelaxations were abolished by all the tested extracts and by the ACh in the presence of L-NAME $(1 \mathrm{mM})$ plus INDO $(1 \mathrm{mM})$, but no significant change was noted in the case of SNP. The results confirmed that the extracts caused partial vasorelaxation through endothelial cells producing NO and prostacyclin $\left(\mathrm{PGI}_{2}\right)$. 
Table 4. Effect of inhibitors on vasorelaxant activity of Spilanthes acmella Murr. extracts.

\begin{tabular}{|c|c|c|c|c|c|c|c|c|}
\hline \multirow{3}{*}{ Compound } & \multicolumn{8}{|c|}{ Vasorelaxant activity } \\
\hline & \multicolumn{2}{|c|}{-Inhibitor } & \multicolumn{2}{|c|}{ +L-NAME (1 mM) } & \multicolumn{2}{|c|}{ +INDO (1 mM) } & \multicolumn{2}{|c|}{$\begin{array}{c}\text { +L-NAME (1 mM) + } \\
\text { INDO (1 mM) }\end{array}$} \\
\hline & $\begin{array}{l}\mathrm{R}_{\max } \\
(\%)\end{array}$ & $\begin{array}{c}\mathrm{ED}_{50} \\
(\mathrm{mg} / \mathrm{mL})\end{array}$ & $\begin{array}{l}\mathbf{R}_{\max } \\
(\%)\end{array}$ & $\begin{array}{c}\mathbf{E D}_{50} \\
(\mathrm{mg} / \mathrm{mL})\end{array}$ & $\mathbf{R}_{\max }(\%)$ & $\begin{array}{c}\mathbf{E D}_{50} \\
(\mathrm{mg} / \mathrm{mL})\end{array}$ & $\mathbf{R}_{\max }(\%)$ & $\begin{array}{c}\mathrm{ED}_{50} \\
(\mathrm{mg} / \mathrm{mL})\end{array}$ \\
\hline $\mathrm{ACh}^{\mathrm{a}}$ & $\begin{array}{c}121.74 \pm \\
1.440\end{array}$ & $9.990 \times 10^{-7}$ & $\begin{array}{c}81.34 \pm \\
0.770\end{array}$ & $5.455 \times 10^{-7}$ & $\begin{array}{c}68.78 \pm \\
0.919\end{array}$ & $4.575 \times 10^{-7}$ & 0 & - \\
\hline $\begin{array}{l}\text { Hexane } \\
\text { extract }^{\mathrm{a}}\end{array}$ & $\begin{array}{c}69.05 \pm \\
0.693\end{array}$ & $5.016 \times 10^{-7}$ & $\begin{array}{c}31.29 \pm \\
0.619\end{array}$ & $3.163 \times 10^{-7}$ & $\begin{array}{c}26.64 \pm \\
0.768\end{array}$ & $3.162 \times 10^{-7}$ & 0 & - \\
\hline $\begin{array}{l}\text { Chloroform } \\
\text { extract }^{\mathrm{a}}\end{array}$ & $\begin{array}{c}94.25 \pm \\
0.873\end{array}$ & $5.278 \times 10^{-7}$ & $\begin{array}{c}51.62 \pm \\
0.706\end{array}$ & $1.598 \times 10^{-6}$ & $\begin{array}{c}46.70 \pm \\
0.511\end{array}$ & $6.661 \times 10^{-7}$ & 0 & - \\
\hline $\begin{array}{l}\text { Ethyl acetate } \\
\text { extract }^{\mathrm{a}}\end{array}$ & $\begin{array}{c}85.84 \pm \\
1.196\end{array}$ & $8.552 \times 10^{-8}$ & $\begin{array}{c}52.95 \pm \\
0.976\end{array}$ & $7.804 \times 10^{-7}$ & $\begin{array}{c}44.48 \pm \\
0.350\end{array}$ & $3.133 \times 10^{-7}$ & 0 & - \\
\hline $\begin{array}{l}\text { Methanol } \\
\text { extract }^{\mathrm{b}}\end{array}$ & $\begin{array}{c}65.56 \pm \\
0.535\end{array}$ & $9.444 \times 10^{-7}$ & $\begin{array}{c}33.24 \pm \\
0.608\end{array}$ & $1.404 \times 10^{-6}$ & $\begin{array}{c}32.19 \pm \\
0.420\end{array}$ & $1.072 \times 10^{-6}$ & 0 & - \\
\hline $\mathrm{SNP}^{\mathrm{b}}$ & $\begin{array}{c}120.84 \pm \\
1.176\end{array}$ & $3.164 \times 10^{-7}$ & $\begin{array}{c}116.70 \pm \\
1.290\end{array}$ & $3.167 \times 10^{-7}$ & $\begin{array}{c}112.93 \pm \\
0.613\end{array}$ & $3.155 \times 10^{-7}$ & $\begin{array}{c}104.98 \pm \\
1.407\end{array}$ & $3.165 \times 10^{-7}$ \\
\hline
\end{tabular}

a: data obtained from 5 experiments, b: data obtained from 6 experiments

$\mathrm{ED}_{50}$ of ACh and SNP are expressed as molar, $p<0.05$, -Inhibitor: in the absence of L-NAME or INDO

Figure 6. Effect of EtOAc extract and ACh on the vascular function of rat thoracic aorta under removal of endothelium (-et) compared with intact endothelium (+et).

\section{EtOAc extract (et)}

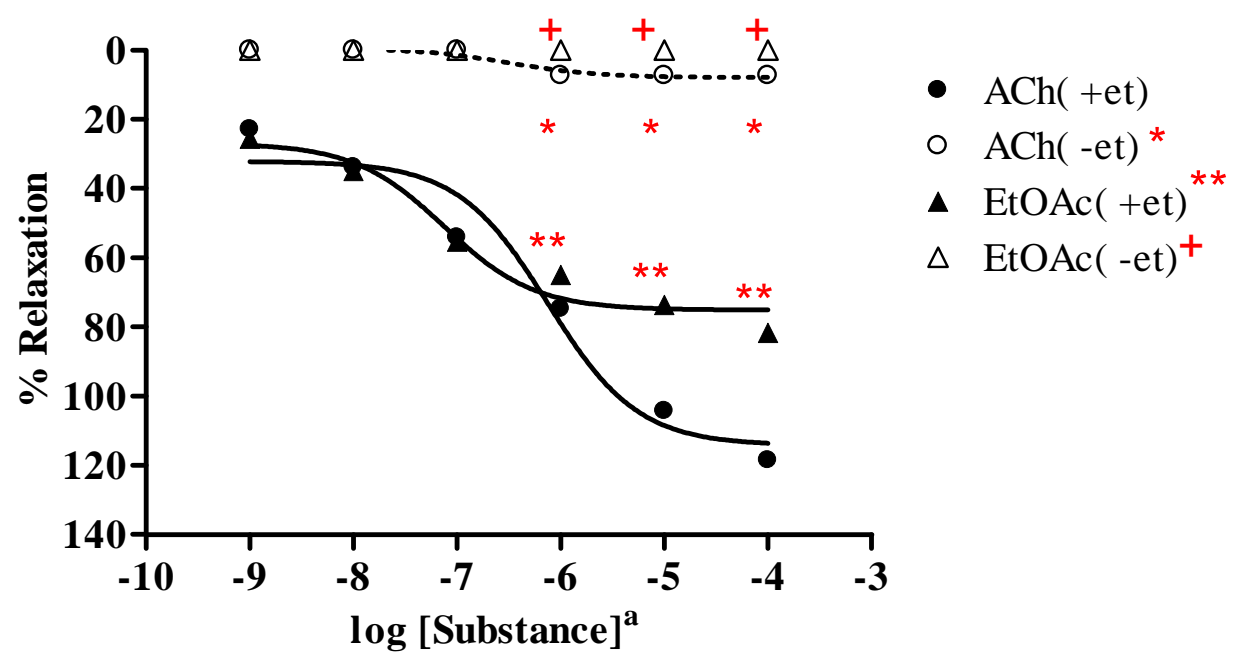

Data represent as means \pm s.e.m. of 4 experiments, each performed in duplicate.

$\star p<0.05, \mathrm{ACh}(+\mathrm{et})$ versus $\mathrm{ACh}(-\mathrm{et}), \star \star x<0.05, \mathrm{ACh}(+\mathrm{et})$ versus EtOAc extract (+et),

$+p<0.05$, EtOAc extract (+et) versus EtOAc extract (-et)

a: EtOAc extract as $\mathrm{mg} / \mathrm{mL}$, ACh as Molar 
Figure 7. Effect of ACh in the presence of L-NAME plus INDO on the vascular function of rat thoracic aorta precontracted with $\mathrm{PE}$.

\section{Ach INDO}

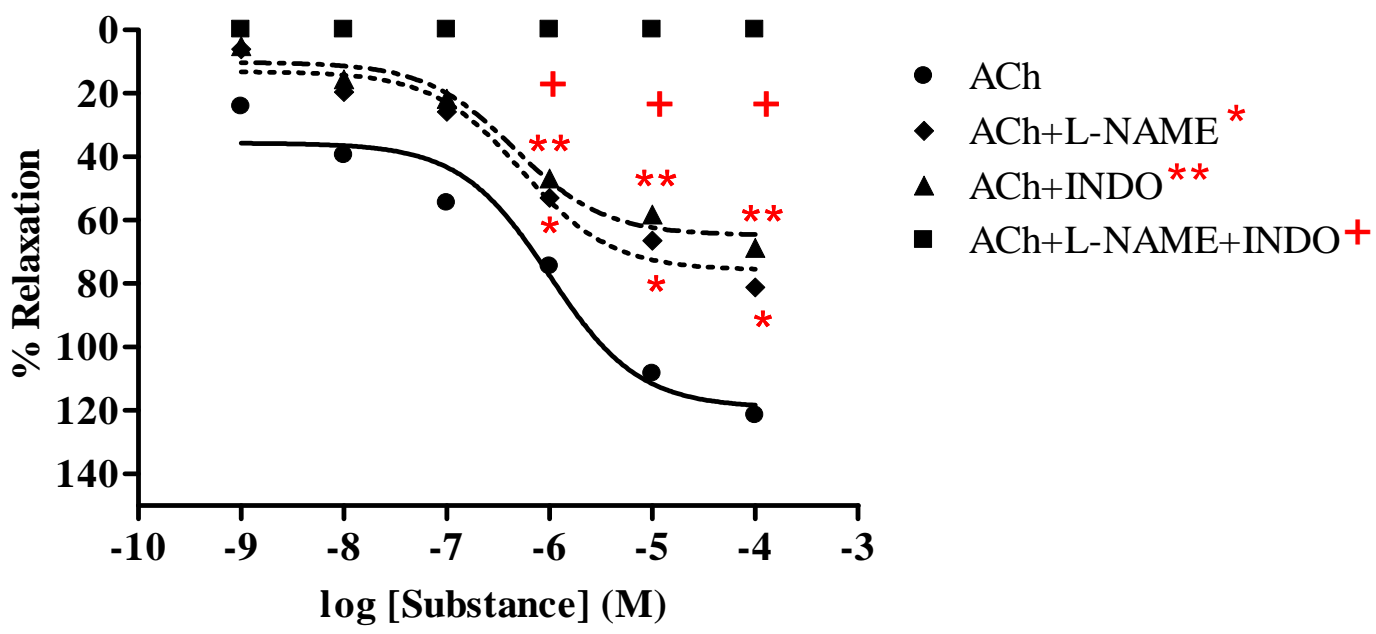

Data represent as means \pm s.e.m. of 5 experiments, each performed in duplicate.

$\star p<0.05$, ACh versus ACh+L-NAME,

$\star \star p<0.05$, ACh versus ACh+INDO,

$+p<0.05$, ACh versus ACh+L-NAME+INDO

Figure 8. Effect of EtOAc extract on the vascular function of rat thoracic aorta in the presence of INDO compared with L-NAME and with L-NAME plus INDO.

\section{EtOAc extract INDO}

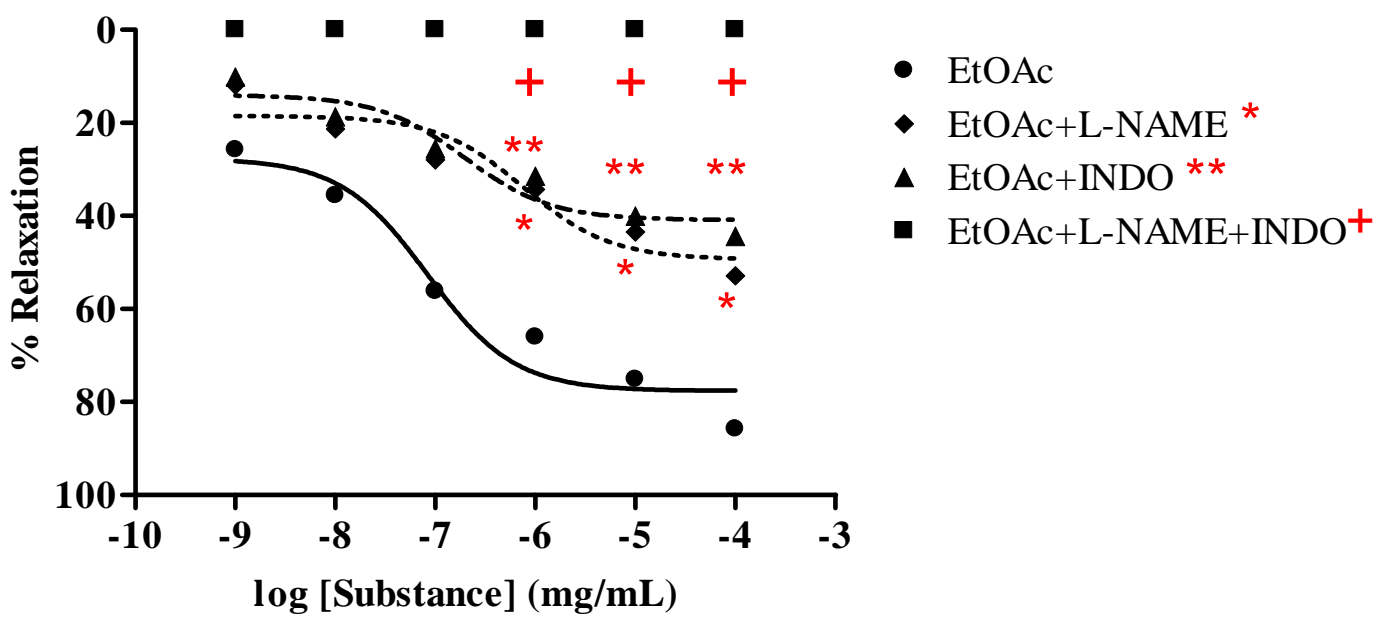

Data represent as means \pm s.e.m. of 5 experiments, each performed in duplicate.

$\star p<0.05$, EtOAc extract versus EtOAc extract+L-NAME,

$\star \star p<0.05$, EtOAc extract versus EtOAc extract+INDO,

$+p<0.05$, EtOAc extract versus EtOAc extract+L-NAME+INDO 
Figure 9. Effect of SNP in the presence of L-NAME, INDO and L-NAME plus INDO on the vascular function of rat thoracic aorta.

\section{SNP INDO}

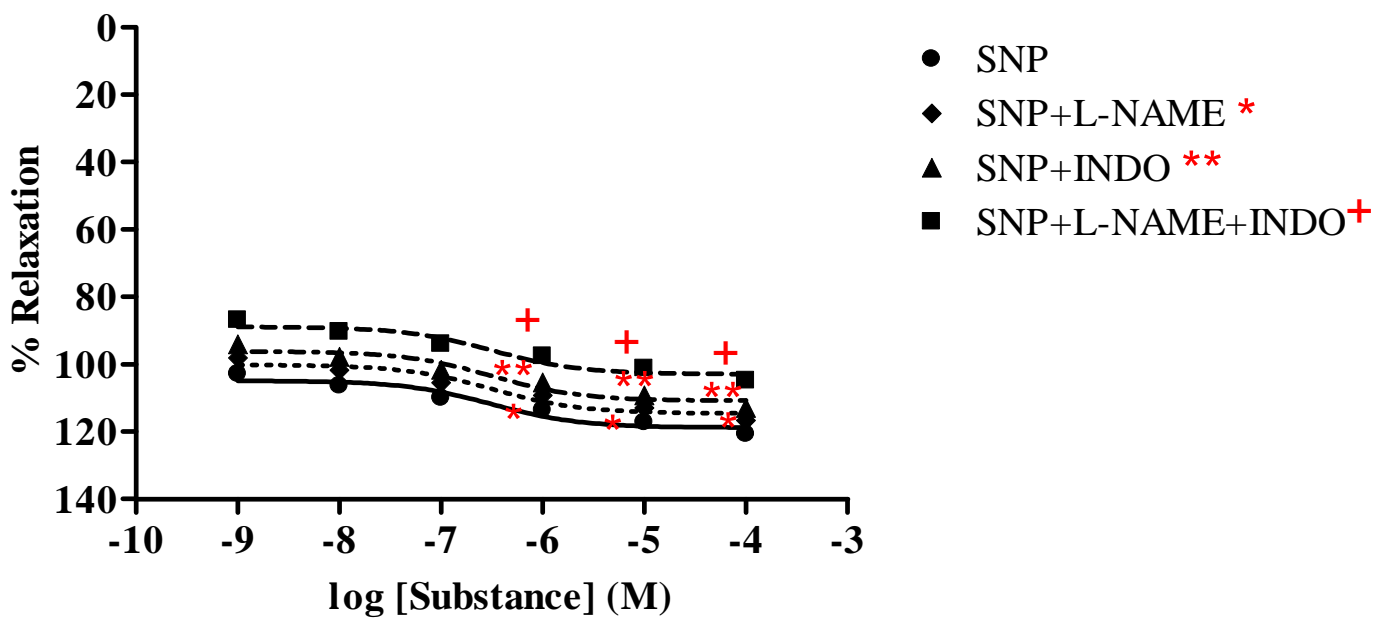

Data represent as means \pm s.e.m. of 6 experiments, each performed in duplicate.

$\star p<0.05$, SNP versus SNP+L-NAME,

$\star \star p<0.05$, SNP versus SNP+INDO,

$+p<0.05, \mathrm{SNP}$ versus SNP+L-NAME+INDO

\subsection{Antioxidant activity}

The antioxidant activity of Spilanthes acmella Murr. extracts was measured using DPPH and SOD assays. The results (Table 5) showed that all the tested extracts exhibited antioxidative activity. In DPPH assay (Figure 10) at $200 \mu \mathrm{g} / \mathrm{mL}$, the ethyl acetate and methanol extracts displayed comparable activity and the highest radical scavenging activity (47.90 and 47.76\%) with IC $_{50} 216$ and $223 \mu \mathrm{g} / \mathrm{mL}$, while $\alpha$-tocopherol (a positive control) showed antioxidant activity with $\mathrm{IC}_{50} 6.67 \mu \mathrm{g} / \mathrm{mL}$. The chloroform extract exhibited $29.82 \%$ radical scavenging activity. The hexane extract produced some activity (4.90\% radical scavenging).

Table 5. Radical scavenging activity and NBT inhibition of extracts.

\begin{tabular}{|c|c|c|}
\hline Extract & $\begin{array}{c}\text { \% Radical scavenging activity } \\
\text { (DPPH assay); } 200 \mu \mathrm{g} / \mathrm{mL}\end{array}$ & $\begin{array}{c}\% \text { NBT inhibition } \\
\text { (SOD assay); } 200 \mu \mathrm{g} / \mathrm{mL}\end{array}$ \\
\hline Hexane & 4.90 & 0.41 \\
\hline Chloroform & 29.82 & 57.92 \\
\hline Ethyl acetate & 47.90 & 33.05 \\
\hline Methanol & 47.76 & 47.02 \\
\hline
\end{tabular}

a: $\alpha$-Tocopherol was used as a positive control.

b: Native SOD (8.1 U) from bovine erythrocytes was used as a positive control. 
The SOD assay at $200 \mu \mathrm{g} / \mathrm{mL}$ (Figure 11), the chloroform extract showed the highest antioxidant activity (57.92\% NBT inhibition). The ethyl acetate and methanol extracts exhibited weak to moderate SOD activity.

Figure 10. Radical scavenging activity of $\alpha$-tocopherol and extracts.

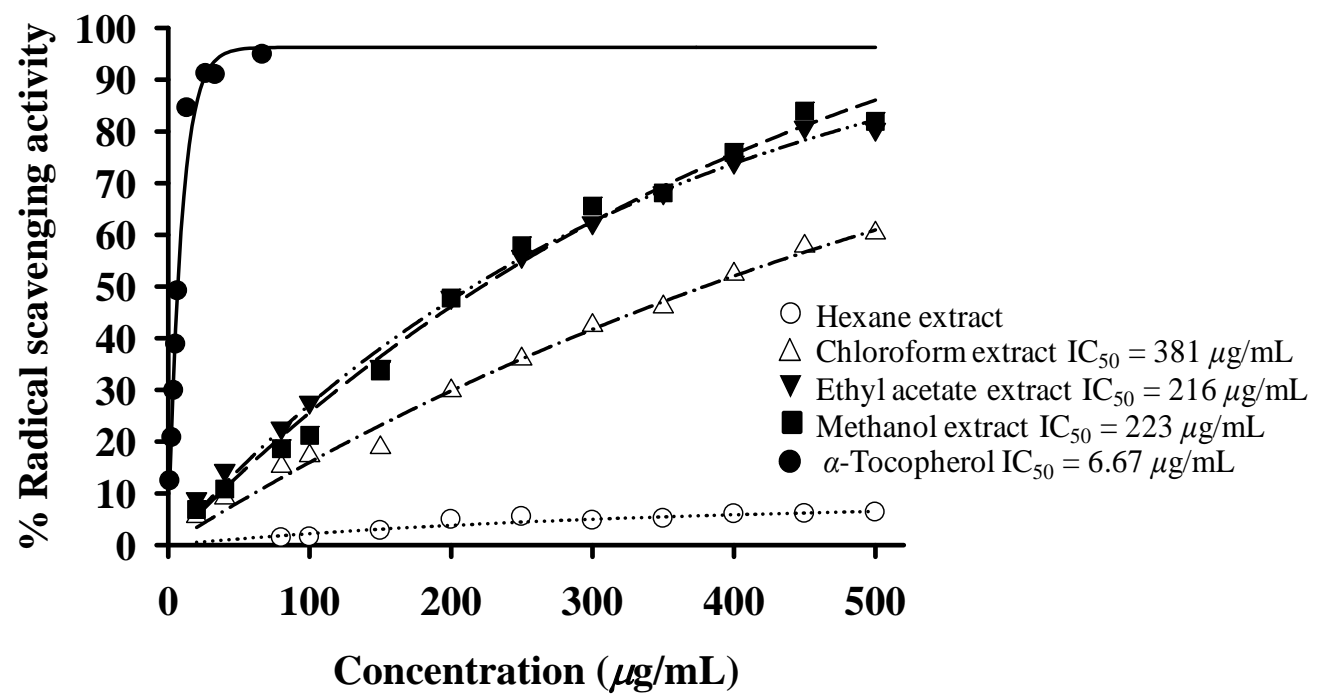

Figure 11. Superoxide dismutase activity of crude extracts.

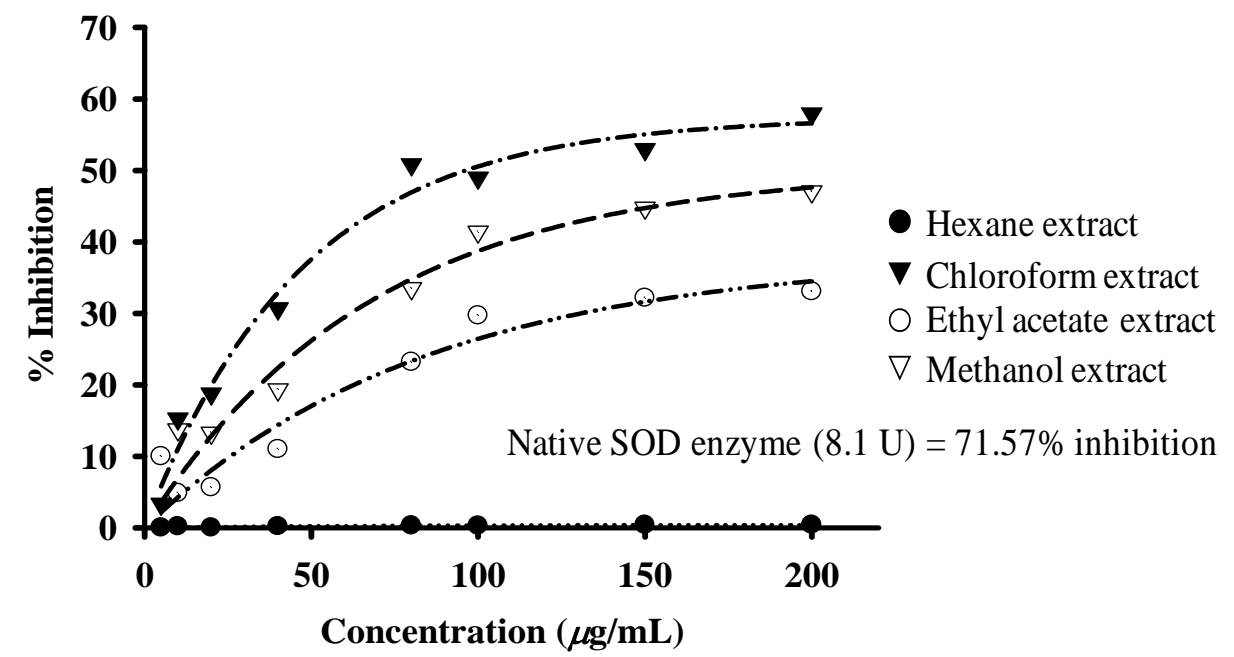

\section{Discussion}

We have disclosed the effect of Spilanthes acmella Murr.extracts on phenylephrine-induced contraction of rat thoracic aorta. All the tested plant extracts elicit maximal vasorelaxations in a doserelated manner, although such vasorelaxations are less than those produced by ACh. The vasorelaxations are exerted by partial production of NO from functional endothelial cells, which is demonstrated by a significant reduction of the activity in the presence L-NAME (Table 2).

The chloroform extract exhibited the highest $\mathrm{R}_{\max }$ of $96.6 \%$, compared to the other extracts. The ethyl acetate extract shows strong vasorelaxation $\left(\mathrm{R}_{\max } 81.6 \%\right)$, while the nonpolar hexane and polar 
methanol extracts exert comparable activity, with $\mathrm{R}_{\max } 65.67$ and $65.09 \%$, respectively. Significantly, the ethyl acetate extract exhibits immediate vasorelaxation with $\mathrm{ED}_{50} 7.61 \times 10^{-8} \mathrm{mg} / \mathrm{mL}$ (Figure 4) when compared to the chloroform extract showing $\mathrm{ED}_{50} 4.28 \times 10^{-7} \mathrm{mg} / \mathrm{mL}$ (Figure 3). This perhaps due to the fact that the ethyl acetate extract has higher affinity for the receptor than the chloroform extract and thus the ethyl acetate extract is the most potent vasorelaxant. Furthermore, the vasorelaxant activities of the tested extracts were all abolished by the removal of functional endothelial cells (Table 3 and Figure 6). This again confirms that the vasorelaxation of the extracts is modulated via NO production by endothelial cells, a fact which is known for Ach involvement in mediating vasorelaxation by $\mathrm{NO}, \mathrm{PGI}_{2}$ and endothelium-derived hyperpolarizing factor [20-22]. Therefore, a set of experiments was studied in the presence of INDO (1 mM) compared with L-NAME (1mM). The results (Table 4) show that the vasorelaxation of the tested extracts (Figure 8) and ACh (Figure 7) is significantly reduced in a dose-dependent manner when compared to that of in the presence of L-NAME. Particularly, the INDO exhibits stronger reduction of such vasorelaxation than the L-NAME, but none was not observed for the SNP (Figure 9). Additionally, in the presence of INDO (1 mM) plus L-NAME (1 mM), synergistic effects are observed, leading to complete loss of the activity of the tested extracts and ACh. However, such an effect is not markedly noticeable for the SNP. These data confirm that the vasorelaxant activity of Spilanthes acmella Murr. is exerted by functional endothelial cells producing partial synthesis of $\mathrm{NO}$ and $\mathrm{PGI}_{2}$ which are inhibited by LNAME and INDO, respectively. NO is an important signaling molecule implicated in cardiovascular functions such as vascular tone. $\mathrm{PGI}_{2}$, endothelium-derived relaxing factor (EDRF), is a powerful vasorelaxant and antioxidant. It exerts antifibrotic properties preventing the development of fibrosis and cirrhosis in liver diseases including antiplatelet aggregation. $\mathrm{PGI}_{2}$ is clinically used for treatment of pulmonary hypertension and portopulmonary hypertension [23].

Many studies have reported the role of NO related to superoxide radical $\left(\mathrm{O}_{2}{ }^{-}\right)$[24]. Thus, the antioxidative activity of the extracts was evaluated. It was found that the plant extracts exhibited antioxidant activity at $200 \mu \mathrm{g} / \mathrm{mL}$. In a DPPH assay, the polar ethyl acetate and methanol extracts exerted the highest radical scavenging activity, while the chloroform extract shows the highest SOD activity. The nonpolar hexane extract produces some activity in both the DPPH and SOD assays.

It is noted that the ethyl acetate extract is the most potent, with immediate vasorelaxation and with the highest radical scavenging activity. This is presumably due to the presence of bioactive polar phenolic and triterpenoid ester compounds in the extract. Moreover, the chloroform extract possesses the highest $\mathrm{R}_{\max }$ accompanied by the highest SOD activity. This may result from the presence of triterpenoids and fatty alcohols or esters in this extract. Moderate vasorelaxations are observed for the nonpolar hexane and polar methanol extracts. This suggests that both hydrophobic and hydrophilic constituents show comparable vasorelaxant activity (Table 2). At this point, the ethyl acetate extract is the most promising vasorelaxant, with a significant $\mathrm{ED}_{50}$ of $76.1 \mathrm{ng} / \mathrm{mL}$ and it is also the most active antioxidant (DPPH assay). It has been reported that flavonoid, phenolic, polyphenol and triterpenoid compounds exhibit both vasorelaxant and antioxidant activities, in some cases behaving as either vasorelaxants or antioxidants [24-26]. Therefore, our results imply a good correlation between vasorelaxant and antioxidant activities deriving from a diverse group of compounds.

It is well recognized that endothelium NO is diminished by reacting with $\mathrm{O}_{2}{ }^{-}$to form peroxynitrite as a potent oxidant, which is inhibited by antioxidant, thus in turn improving NO induced 
vasorelaxation [24, 27-29]. This was evidenced by phenolic antioxidants such as prinsepiol isolated from roots of Valeriana prionophylla [24]. A number of flavonoids exerted vasorelaxation through endothelium produced NO, e.g. chrysin (5, 7-dihydroxyflavone) presented in honey, propolis, fruits, vegetables and beverages [30-32], isoliquiritigenin isolated from various plants such as Dahlia variabilis [33] and recently from Hydnophytum formicarum Jack. [34]. Triterpenoids are commonly found in plant species, for instances, oleanolic acid and erythrodiol elicit vasorelaxation via endothelium induced NO [26]. In addition, root extracts of Caesalpinia benthamiana, which are rich in phenolic compounds, e.g. gallic acid, resveratrol and tannin, had significant vasorelaxant and powerful scavenging activity versus $\mathrm{O}_{2}{ }^{-}$[25]. Some plant extracts of Casimiroa edulis and Mammea africana (Guttiferae) caused vasorelaxation through endothelium dependent producing NO [35, 36]. The vasorelaxation was also mediated by both endothelium dependent and endothelium independent pathways [37]. Moreover, extracts of ethnomedical plant used as antihypertensive and anti-stroke exhibited their vasorelaxants via endothelium dependent producing NO or via direct stimulation of NO release. Examples are Diospyros kaki Thunb., Polygonum aviculare L., Magnolia liliflora Desr., Sorbus commixta Hedl, Selaginella tamariscina Spr. and Guazuma ulmifolia [38, 39].

In this study, Spilanthes acmella Murr. extracts produce vasorelaxation via partial release of NO and $\mathrm{PGI}_{2}$ from endothelium as well as showing antioxidant activities. Some countries in Asia including Thailand use this plant as fresh vegetable and cooking ingredient [40]. This demonstrates beneficial effects and applications of the Spilanthes acmella Murr. to medical uses and as a health food.

\section{Experimental Section}

\subsection{Materials}

\subsubsection{Chemicals}

L-Phenylephrine hydrochloride, sodium nitroprusside, $N^{\mathrm{G}}$-nitro-L-arginine methyl ester, acetylcholine, ketamine hydrochloride and indomethacin were obtained from Sigma Chemical Co. (St. Louis, MO, USA). Polyethyleneglycol was purchased from Fluka. Plant extracts were dissolved in PEG, while methanol extract was dissolved in $0.9 \%$ normal saline. Then the solutions were further diluted by normal saline.

\subsubsection{Plant materials}

Aerial parts of Spilanthes acmella Murr. were collected from Nakornsrithammarat Province, Thailand. It has been identified (BKF 112361) by The Forest Herbarium, Royal Forestry Department. A voucher specimen has been deposited at Department of Chemistry, Faculty of Science, Srinakharinwirot University, Thailand. 


\subsection{Methods}

\subsubsection{Extraction}

The air dried Spilanthes acmella Murr. (1,050 g) was ground and extracted with hexane (3×5 days), followed by filtration. The filtrates were combined and evaporated in vacuo to give a crude hexane extract $(11 \mathrm{~g})$. Similarly, the extraction was performed using chloroform, ethyl acetate and methanol to obtain the corresponding chloroform (10 g), ethyl acetate (18 g) and methanol (31 g) extracts, respectively.

\subsubsection{Components and HPLC profiles of the extracts}

TLC chromatograms of the extracts were run on pre-coated silica gel $60 \mathrm{~F}_{254}$ (Merck). Phenolic groups were detected by UV fluorescence. Their IR (neat, Perkin Elmer Spectrum One FT-IR spectrometer) and ${ }^{1} \mathrm{H}-\mathrm{NMR}\left(\mathrm{CDCl}_{3}\right.$ or DMSO- $\mathrm{d}_{6}$, Bruker Avance $300 \mathrm{MHz}$ ) spectra were recorded. HPLC profiles were performed with flow rate $1 \mathrm{~mL} / \mathrm{min}$ using a Hichrom Exsil 100-5ODS column (25 cm length with $4.6 \mathrm{~mm}$ diameter) and a Waters 2996 Photodiode Array Detector. The tested extracts were prepared by dissolving $1 \mathrm{mg}$ in $1 \mathrm{~mL}$ methanol. Aliquots of $20 \mu \mathrm{L}$ were injected using gradient elution with decreasing polarity from $\mathrm{H}_{2} \mathrm{O}: \mathrm{CH}_{3} \mathrm{OH}$ (9:1) to $\mathrm{CH}_{3} \mathrm{OH}$ and running for $100 \mathrm{~min}$. Peaks of components were detected at $228.0 \mathrm{~nm}$ (hexane, chloroform and ethyl acetate extracts) and at $280.0 \mathrm{~nm}$ (methanol extract).

\subsubsection{Biological activity}

The study was performed using the hexane, chloroform, ethyl acetate and methanol extracts of Spilanthes acmella Murr., obtained as described above.

\subsubsection{Vasorelaxant assay}

\subsection{Isometric tension measurements}

The protocols for handling animals were approved by the Animal Care Committee at the Srinakharinwirot University and done at the National Laboratory Animal Centre, Mahidol University. Male Sprague-Dawley rats (170-250 g) were anesthetized with intraperitoneal ketamine hydrochloride $(0.05 \mathrm{~mL} / \mathrm{kg})$. The thoracic aorta was quickly removed to cold Kreb-Henseleit buffer containing (mM): $118 \mathrm{NaCl} ; 4.7 \mathrm{KCl} ; 1.2 \mathrm{KH}_{2} \mathrm{PO}_{4} ; 1.2 \quad \mathrm{MgSO}_{4} \cdot 7 \mathrm{H}_{2} \mathrm{O} ; 11.0(+)$-glucose; $25.0 \mathrm{NaHCO}_{3}$; and 2.5 $\mathrm{CaCl}_{2} \cdot 2 \mathrm{H}_{2} \mathrm{O}, \mathrm{pH} 7.4$, aerated with $95 \% \mathrm{O}_{2}, 5 \% \mathrm{CO}_{2}$. After removed debris tissue, the vessel was cut into rings, each 2-3 mm-long and hanged in the organ bath containing Kreb-Henseleit solution at $37{ }^{\circ} \mathrm{C}$, aerated with $95 \% \mathrm{O}_{2}, 5 \% \mathrm{CO}_{2}$ and also connected to a force-displacement transducer (Model MLTO50 Force transducer Range: 50, P.R. China) and equilibrated for 50-60 min under a $1 \mathrm{~g}$ resting tension. During an incubation period, the Kreb-Henseleit solution was changed every $20 \mathrm{~min}$. After the incubation period, the maximal contraction of the rings was determined with high dose of PE $\left(10^{-5} \mathrm{M}\right)$ 
and then washed 5 times until resting tension was recovered. Isometric tension [41] was recorded by Macintosh MacLab 4E AD Instrument connected to computer hard drive. The endothelial intact was examined using high dose of ACh $\left(10^{-5} \mathrm{M}\right)$ at a level of submaximal tension. If the relaxation response to ACh was less than $80 \%$, the ring would be discarded. Then, the ring was washed 5 times to remove the residue of ACh. The vessel was again equilibrated for 50-60 min and the responses of vessel were performed by the following protocols. Submaximal contraction was induced using PE $\left(10^{-7} \mathrm{M}\right)$, then cumulative dose-response curves to the agonists $\left(10^{-9}-10^{-4} \mathrm{M}\right.$ or $\left.\mathrm{mg} / \mathrm{mL}\right)$. Finally, the dose-response curve of SNP was performed in order to test the functional vessel. With inhibitors (L-NAME or INDO) or vehicle, the vessels were pretreated with such compounds prior to submaximal contraction with PE then examine the endothelial response to the tested compounds. After each cumulative doseresponse curve, the thoracic aorta preparation was washed and equilibrated 50-60 min before working on the next dose-response curve of tested compounds.

\subsection{Statistical analyses}

The unpaired two-tailed Student's t test and one-way ANOVA were used in the statistical analysis when appropriate. Post-hoc comparisons of individual groups were performed using the TukeyKramer test. The $\mathrm{ED}_{50}$ values for the vasorelaxants were calculated using nonlinear regression analyses (GraphPad Prism 4, GraphPad Software Inc., Sandiego, CA, USA). A p-value less than 0.05 was considered significant. The data were expressed as mean \pm s.e.m. for the number of animals.

\subsubsection{Antioxidative assay}

Two assay methods were used: 2,2-diphenyl-1-picrylhydrazyl (DPPH) and superoxide dismutase (SOD). The antioxidative activity of the crude extracts was elucidated by the DPPH radical scavenging assay [42]. When DPPH (a stable purple color) reacts with an antioxidant, it is reduced to yield a lightyellow colored diphenylpicrylhydrazine. Color changes can be spectrophotometrically measured. In this study, experiment was initiated by preparing $0.2 \mathrm{mM}$ solution of DPPH in methanol. One $\mathrm{mL}$ of this solution was added sample solution $(1 \mathrm{mg} / \mathrm{mL}$ dissolved in methanol, $0.5 \mathrm{~mL})$. After $30 \mathrm{~min}$, absorbance was measured at $517 \mathrm{~nm}$ and the percentage of radical scavenging activity was calculated from the following equation:

$$
\% \text { Radical scavenging }=(1 \text {-Abs.sample/Abs.cont }) \times 100
$$

where Abs.cont is the absorbance of the control reaction and Abs.sample is the absorbance in the presence of sample.

The SOD activity was assayed by measuring inhibition of the photoreduction of nitro blue tetrazolium (NBT) [43]. The indirect assay is comprised of several reactions: the photochemically excited riboflavin was first reduced by methionine into a semiquinone, which donated an electron to oxygen to form the superoxide source. The superoxide readily converted NBT into a purple formazan product. In this regard, the SOD activity was inversely related to the amount of formazan formation. 


\section{Conclusions}

Our findings report for the first time that Spilanthes acmella Murr. extracts possess vasorelaxant and antioxidant activities. The plant extracts elicit vasorelaxations via partially endothelium induced $\mathrm{NO}$ and $\mathrm{PGI}_{2}$ in a dose-dependent manner. However, other underlying mechanisms may participate. Significantly, the ethyl acetate extract exhibits immediate vasorelaxation in nanogram levels and is the most potent antioxidant in the DPPH assay. The chloroform extract displays the highest vasorelaxation with the highest antioxidant (SOD assay). Furthermore, the nonpolar hexane and polar methanol extracts show moderate vasorelaxant activity. This demonstrates an important role for Spilanthes acmella Murr. as a new natural source of vasodilators and antioxidants. Furthermore, the results provide a guideline to further isolate bioactive ingredient, essentially, from chloroform and ethyl acetate extracts. However, the methanol and hexane extracts are also interesting to explore. Hopefully, new constituents will be isolated and investigated for bioactivities. Particularly, mechanism of vasorelaxation in more details involved EDHF via different potassium channels will be investigated.

\section{Acknowledgements}

This project was in part supported by the budget grant of Srinakharinwirot University (B.E. 2549) and the annual research grant of Mahidol University (B.E. 2551-2555). We thank Dr. Vilailak Prachyawarakorn, Chulabhorn Research Institute, for the technical support on the HPLC profiles determination.

\section{References}

1. Chopra, R.N.; Nayara, S.L.; Chopra, I.C. Glossary of Indian medicinal plants; Council of Scientific \& Industrial Research: New Delhi, 1956; pp. 168-169.

2. Bunyapraphatsara, N.; Chokechareunporn, O. Tradition medicinal plants; Prachachon: Bangkok, 1999.

3. Farnsworth, N.R.; Bunyapraphatsara, N. Thai medicinal plants recommended for primary health care system; Prachachon: Bangkok, 1992.

4. Perry, L.M.; Metzger, J. Medicinal plants of East and South-East Asia; MIT Press: Cambridge, 1980.

5. Wilkinson, J. Herbs and flowers of the cottage garden; Book Builders: Melbourne, 1989.

6. Gokhale, V.G.; Bhide, B.V. Chemical investigation of Spilanthes acmella. J. Ind. Chem. Soc. 1945, 22, 250-252.

7. Ramsewak, R.S.; Erickson, A.J.; Nair, M.G. Bioactive $N$-isobutylamides from the flower buds of Spilanthes acmella. Phytochemistry 1999, 51, 729-732.

8. Krishnaswamy, N.R.; Prasanna, S. $\alpha$ and $\beta$-Amyrin esters and sitosterol glucoside from Spilanthes acmella. Phytochemistry 1975, 14, 1666-1667.

9. Mukharya, D.K.; Ansari, A.H. Olean-12-en-3-O-beta-D-galactopyranosyl (1 $\rightarrow$ 4)-O-alpha-Lrhamnopyranoside: A new triterpenoidal saponin from the roots of Spilanthes acmella (Murr.). Indian J. Chem. B 1987, 26, 86. 
10. Saengsirinavin, C.; Vanveerakul B.; Chalermsanyakorn, P. Local anesthetic action of Spilanthes acmella Murr. Siriraj Hospital Gazazette 1990, 42, 123-131.

11. Ansari, A.H.; Mukharya, D.K.; Saxena, V.K. Analgesic study of N-isobutyl-4,5-decadienamide isolated from the flowers of Spilanthes acmella (Murr). Indian J. Pharm. Sci. 1988, 50, 106.

12. Ratnasooriya, W.D.; Pieris, K.P.P. Attenuation of persistent pain and hyperalgesia by Spilanthus acmella flowers in rats. Pharm. Biol. 2005, 43, 614-619.

13. Saraf, D.K.; Dixit, V.K. Spilanthes acmella Murr.: Study on its extract spilanthol as larvicidal compound. Asian J. Exp. Sci. 2002, 16, 9-19.

14. Pitasawat, B.; Choochote, W.; Kanjanapothi, D.; Panthong, A.; Jitpakdi, A.; Chaithong, U. Screening for larvicidal activity of ten carminative plants. Southeast Asian J. Trop. Med. Public Health 1998, 29, 660-662.

15. Ekanem, A.P.; Wang, M.; Simon, J.E.; Moreno, D.A. Antiobesity properties of two African plants (Afromomum meleguetta and Spilanthes acmella) by pancreatic lipase inhibition. Phytother. Res. 2007, 21, 1253-1255.

16. Ratnasooriya, W.D.; Pieris, K.P.P.; Samaratunga, U.; Jayakody, J.R.A.C. Diuretic activity of Spilanthes acmella flowers in rats. J. Ethnopharmacol. 2004, 91, 317-320.

17. Pandey, H.K.; Rawut, P.S.; Kumar, N.; Gauri, S. A herbal formulation for toothache and related disorders and a process for preparation thereof. IN Patent 2004DE00260, Chem. Abstr. 2007, 147, 350526.

18. Adler, R.J. Compositions for the acute and/or long term treatment of periodontal diseases using herb extracts. Eur Patent WO 2006059196, Chem. Abstr. 2006, 145, 14791.

19. Bajarang, B.B. Invention of herbal drug against HIV infection/AIDS. IN Patent 2005MU01512, Chem. Abstr. 2007, 147, 413250.

20. Quignard, J.F.; Félétou, M.; Thollon, C.; Vilaine, J.P.; Duhault, J.; Vanhoutte, P.M. Potassium ions and endothelium-derived hyperpolarizing factor in guinea-pig carotid and porcine coronary arteries. Br. J. Phrmacol. 1999, 127, 27-34.

21. Ferrer, M.; Marin, J.; Encabo, A.; Alonso, M.J.; Balfagón, G. Role of $\mathrm{K}^{+}$channels and sodium pump in the vasodilatation induced by acetylcholine, nitric oxide and cyclic GMP in the rabbit aorta. Gen. Pharmacol. 1999, 33, 35-41.

22. Edwards, G.; Dora, K.A.; Gardener, M.J.; Garland, C.J.; Weston, A.H. $\mathrm{K}^{+}$is an endotheliumderived hyperpolarizing factor in rat arteries. Nature 1998, 396, 269-272.

23. Zardi, E.M.; Dobrina, A.; Amoroso, A.; Afeltra, A. Prostacyclin in liver disease: A potential therapeutic option. Expert Opin. Biol .Ther. 2007, 7, 785-790.

24. Piccinelli, A.L.; Arana, S.; Caceres, A.; Bianca, R.E.V.; Sorrentino, R.; Rastrelli, L. New lignans from the roots of Valeriana prionophylla with antioxidative and vasorelaxant activities. J. Nat. Prod. 2004, 67, 1135-1140.

25. Zamblé, A.; Martin-Nizard, F.; Sahpaz, S.; Hennebelle, T.; Staels, B.; Bordet, R.; Duriez, P.; Brunet, C.; Bailleul, F. Vasoactivity, antioxidant and aphrodisiac properties of Caesalpinia benthamiana roots. J. Ethnopharmacol. 2008, 116, 112-119.

26. Rodríguez-Rodríguez, R.; Herrera, M.D.; Perona J.S.; Ruiz-Gutiérrez, V. Potential vasorelaxant effects of oleanolic acid and erythrodiol, two triterpenoids contained in 'orujo' olive oil, on rat aorta. Br. J. Nutr. 2004, 92, 635-642. 
27. Demirci, B.; McKeown, P.P.; Bayraktutan DVM, U. The bimodal regulation of vascular function by superoxide anion: Role of endothelium. BMB rep. 2008, 41, 223-229.

28. Shin, J.I.; Lee, Y.K.; Kim, Y.M.; Hwang, J.T.; Park, O.J. Possible link between NO concentrations and COX-2 expression in systems treated with soy-isoflavones. Ann. N. Y. Acad. Sci. 2007, 1095, 564-573.

29. Zenebe, W.; Pechánová, O.; Andriantsitohaina, R. Red wine polyphenols induce vasorelaxation by increased nitric oxide bioactivity. Physiol. Res. 2003, 52, 425-432.

30. Villa, I.C.; Vera, R.; Galisteo, M.; O’Valle, F.; Romero, M.; Zarzuelo, A.; Duarte, J. Endothelial nitric oxide production stimulated by bioflavonoid chrysin in rat isolated arota. Planta. Med. 2005, 71, 829-834.

31. Norton, C.; Kalea, A.Z.; Harris, P.D.; Klimis-Zacas, D.J. Wild blueberry-rich diets affect the contractile machinery of the vascular smooth muscle in the Sprague-Dawley rat. J. Med. Food 2005, 8, 8-13.

32. DalBó, S.; Moreira, E.G.; Brandão, F.C.; Horst, H.; Pizzolatti. M.G.; Micke, G.A.; Ribeiro-doValle, R.M. Mechanisms underlying the vasorelaxant effect induced by proanthocyanidin-rich fraction from Croton celtidifolius in rat small resistance arteries. J. Pharmacol. Sci. 2008, 106, 234-241.

33. Bate-Smith, E.C.; Swain, T. The isolation of 2,4,4'-trihydroxychalcone from yellow varieties of Dahlia variabilis. J. Chem. Soc. 1953, 2185-2187.

34. Prachayasittikul, S.; Buraparuangsang, P.; Worachartcheewan, A.; Isarankura-Na-Ayudhya, C.; Ruchirawat, S.; Prachayasittikul, V. Antimicrobial and antioxidative activities of bioactive constituents from Hydnophytum formicarum Jack. Molecules 2008, 13, 904-921.

35. Baisch, A.L.M.; Urban, H.; Ruiz, A.N. Endothelium-dependent vasorelaxing activity of aqueous extracts of lyophilized seeds of Casimiroa edulis (AECe) on rat mesenteric arterial bed. $J$. Ethnopharmacol. 2004, 95, 163-167.

36. Dongmo, A.B.; Azebaze, A.G.B.; Nguelefack, T.B.; Ouahouo, B.M.; Sontia, B.; Meyer, M.; Nkengfack, A.E.; Kamanyi, A.; Vierling, W. Vasodilator effect of the extracts and some coumarins from the stem bark of Mammea africana (Guttiferae). J. Ethnopharmacol. 2007, 111, 329-334.

37. Jiang, H.D.; Cai, J.; Xu, J.-H.; Zhou, X.-M.; Xia, Q. Endothelium-dependent and direct relaxation induced by ethyl acetate extract from Flos Chrysanthemi in rat thoracic aorta. J. Ethnopharmacol. 2005, 101, 221-226.

38. Yin, H.H.; Kang, D.G.; Choi, D.H.; Kwon, T.O.; Lee, H.S. Screening of vasorelaxant activity of some medicinal plants used in Oriental medicines. J. Ethnopharmacol. 2005, 99, 113-117.

39. Magos, G.A.; Mateos, J.C.; Páez, E.; Fernández, G.; Lobato, C.; Márquez, C.; Enríquez, R.G. Hypotensive and vasorelaxant effects of the procyanidin fraction from Guazuma ulmifolia bark in normotensive and hypertensive rats. J. Ethnopharmacol. 2008, 117, 58-68.

40. Siemonsma, J.S.; Piluck, K. Plant resources of South East Asia No 8 Vegetable; Pudoc Scientific Publishers: Wageningen, The Netherlands, 1993.

41. Woodman, O.L.; Wongsawatkul, O.; Sobey, C.G. Contribution of nitric oxide, cyclic GMP and $\mathrm{K}^{+}$ channel to acetylcholine induced dilation of rat conduit and resistance arteries. Clin. Exp. Pharmacol. Toxicol. 2000, 27, 32-34. 
42. Prachayasittikul, S.; Suksrichavalit, T.; Isarankura-Na-Ayudhya, C.; Ruchirawat, S.; Prachayasittikul, V. Antimicrobial and antioxidative activities of 1-Adamantylthio derivatives of 3-substitued pyridines. EXCLI J. 2008, 7, 63-70.

43. Piacham, T.; Isarankura-Na-Ayudhya, C.; Nantasenamat, C.; Yainoy, S.; Ye, L.; Prachayasittikul, V. Metalloantibiotic Mn(II)-bacitracin complex mimicking manganese superoxide dismutase. Biochem. Biophys. Res. Comm. 2006, 341, 925-930.

(C) 2008 by the authors; licensee Molecular Diversity Preservation International, Basel, Switzerland. This article is an open-access article distributed under the terms and conditions of the Creative Commons Attribution license (http://creativecommons.org/licenses/by/3.0/). 